\section{Hodentorsion schränkt spätere Fertilität offenbar nicht ein}

Gielchinsky I et al. Pregnancy Rates after Testicular Torsion. J Urol 2016; 196: 852 - 855

Einige Autoren sind der Ansicht, dass eine frühere Hodentorsion die Fertilität des betroffenen Mannes in der Zukunft einschränken könnte. Grundlage dafür sollen kongenitale Abnormitäten der Hoden sein, die überhaupt erst zu der Torsion führen. Darüber hinaus könnten rezidivierende Ischämien und Autoimmunmechanismen eine Rolle spielen. Harte Beweise für die Fertilitätsminderung und ggf. ihre Grundlagen gibt es jedoch nicht.

Mediziner des Hadassah Medical Center in Jerusalem sind daher der Frage systematisch nachgegangen. Dazu haben sie haben insgesamt 63 Männer in eine retrospektive Studie aufgenommen und mit einem standardisierten Fragebogen interviewt.

Die Teilnehmer waren zwischen 1994 und 2014 wegen einer unilateralen Hodentorsion mit bis zu $96 \mathrm{~h}$ bestehender Symptomatik behandelt worden. Dabei war entweder eine einseitige Orchiektomie plus Orchidopexie des kontralateralen Hodens $(n=23)$ oder eine beidseitige Orchidopexie $(n=41)$ vorgenommen worden. Die Indikation zur Orchiektomie war gestellt worden, wenn 20 min nach Detorquierung keine Farbveränderung des Hodens nachweisbar war und nach Inzision der Tunica albuginea keine Blutung auftrat.

Für die Teilnahme an der jetzigen Untersuchung mussten die Männer $\geq 25$ Jahre alt sein, seit > 12 Monaten in einer festen Beziehung leben, und bei den Paaren musste ein Kinderwunsch bestehen. Patienten mit primärer Infertilität und/ oder (Zustand nach) Varikozele und/ oder Maldescensus testis waren ausgeschlossen.

Jetzt wurden die Männer gefragt,
- ob sie Kinder hatten und ggf. wie viele,

- ob die Kinder vor oder nach der Torsion geboren worden waren,

- wie lange es bis zum Eintreten einer Schwangerschaft gedauert hatte

- und ob die Hoden bei der Selbstuntersuchung symmetrisch im Skrotum tastbar waren.

Die Rate der Schwangerschaft betrug insgesamt $90,9 \%$ in der Orchiektomiegruppe bzw. 90,2\% in der Orchidopexiegruppe, die der Lebendgeburten 87,8\% bzw. $86,3 \%$ (jeweils $p=1,0$ ). Diese Raten waren damit denen vergleichbar, die in der internationalen Fachliteratur für gesunde Paare beschrieben sind (82-92\%). Die Dauer bis zum Eintreten der Schwangerschaft betrug 6,6 Monate bzw. 7,2 Monate.

Bei 6 der 63 Männer bestand eine Infertilität $(9,5 \% ; n=2$ in der Orchiektomiegruppe und $n=4$ in der Orchidopexiegruppe). Bei 2 Männern nach Orchidopexie war laut Selbstuntersuchung der betroffene Hoden atrophiert, bei 1 Mann lagen symmetrische Hoden vor, der 4. wollte die Frage nicht beantworten. Die gynäkologische Abklärung ihrer Partnerinnen ergab jeweils einen unauffälligen Befund. Bei insgesamt 3 dieser 6 Paare wurde erfolgreich eine In-vitro-Fertilisation vorgenommen (2 Männer nach Orchiektomie, 1 Mann nach Orchidopexie).

\section{FAZIT}

Eine Hodentorsion scheint also im späteren Alter nicht mit einer eingeschränkten Fertilität verbunden, meinen die Autoren - die von den Partnerschaften der vorgestellten Männer berichteten Schwangerschafts- und Lebendgeburtenraten entsprechen denen der Allgemeinbevölkerung. Einschränkend gilt, das keine weitere Abklärung der Infertilität erfolgte, insbesondere wurde keine Spermaanalyse vorgenommen.

Dr. Elke Ruchalla Bad Dürrheim

\section{Kommentar}

Belastbare Untersuchungen zur Fertilität nach Torsion oder anderen Hodenerkrankungen sind naturgemäß nicht Gegenstand der Kinder- und Jugendmedizin. So stützt sich auch eine aktuelle Leitlinie bei der Zusammenfassung prognostischer Daten nach Hodentorsion auf Untersuchungen Erwachsener, die, vergleichbar dieser Arbeit, z. T. älteren Datums sind, aber anderen Quellen entspringen. Danach scheint die verringerte Gesamtmenge an Keimepithel der eigentliche Faktor zu sein [1,2]. Die resultierende Subfertilität wurde in 36$39 \%$ von Patienten nach Hodentorsion beschrieben; normale Untersuchungsbefunde des Ejakulates fanden sich in weniger als der Hälfte der Fälle im LangzeitFollow-up [1]. In Vergleichen mit der Normalpopulation waren Hinweise auf temporär oder permanent erhöhte Spiegel an Auto-Antikörpern gegen Spermien nach durchgemachter Torsion widersprüchlich und am Ende statistisch nicht signifikant; gleiches gilt für Untersuchungen zum Hormonstatus (FSH, LH und Testosteron) [2,4,5]. Aufgrund der geringen Inzidenz der Hodentorsion und des zu berücksichtigenden Salvage-Effektes durch zeitgerechte Detorsion wurde der durch sie bedingte Anteil an der männlichen Infertilität letztlich mit unter $1 \%$ angegeben [6].

Die Daten der vorliegenden Studie scheinen dies zu bestätigen, indem sich die Rate einer in fester Partnerschaften realisierten Schwanger- (oder besser?) Vaterschaft sowohl nach einseitiger Orchiektomie als auch nach Detorsion und Pexie eines torquierten Hodens nicht von der Normalpopulation unterscheidet. Berücksichtigt man religiöse und soziale Argumente der Autoren gegen etablierte Untersuchungen auf der Basis von Spermiogrammen, so scheint mit der Schwangerschaftsrate ein alternativer, wenig invasiver Parameter gefunden. Sind die Aussagen jedoch vergleichbar? Bei kleiner Fallzahl fallen bereits $80 \%$ der Patienten heraus, die zu jung und/oder nicht verheiratet sind bzw. nicht in einer festen Partnerschaft leben. Die zunächst bei ca. $10 \%$ der verbleibenden Kohorte ausbleibende Vaterschaft kann am Ende 
durch In-vitro-Fertilisation der Partnerinnen realisiert werden. Dem gehen, wie auch bei der anderen Hälfte vermutlich infertiler Patienten, Spermiogramme voraus, die die tatsächliche Fertilität beurteilen.

Der von den Autoren gut begründete Weg, die Schwangerschaft der Partnerin als Indikator für die männliche Fertilität zu wählen, benötigt offensichtlich einen langen Atem, was die Bildung ausreichend große Kohorten mit statistischer Aussagekraft betrifft. Und sie verzichtet am Ende doch nicht auf das Spermiogramm. Man wünschte sich Daten von höherer Vorhersagekraft, um die Fertilität der zahlenmäßig begrenzten Gruppe von Patienten nach Hodentorsion besser zu erfassen. Dass entsprechende Parameter existieren, zeigen aktuelle Arbeiten aus der Reproduktionsmedizin [7]. Auch ist die Bewertung einer Subfertilität mit Blick auf den realisierten Kinderwunsch im Fluss. Solche systematische Nachuntersuchungen wären nicht nur von individuellem Interesse betroffener Patienten. Im Rahmen des Akutereignisses einer Torsion könnten die Ergebnisse auch helfen, die Dringlichkeit aller Bemühungen um einen Hodenerhalt zu untermauern.

Der Autor

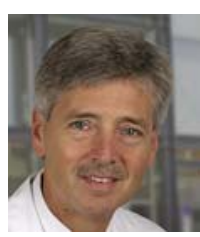

Prof. Dr. med. Christian Lorenz, Klinik für Kinderchirurgie und -urologie, Klinikum Bremen-Mitte

\section{Literatur}

[1] Ferreira U et al. Comparative study of the fertility potential of men with only one testis. Scand J Urol Nephrol 1991; 25: 255-159

[2] Schütte B et al. Exocrine and endocrine testicular function following unilateral torsion - a retrospective clinical study of 36 patients. Urologe A 1986; 25: 142-146

[3] Visser A], Heyns CF. Testicular function after torsion of the spermatic cord. BJU Int 2003; 92: $200-203$

[4] Arap MA et al. Late hormonal levels, semen parameters, and presence of antisperm anti- bodies in patients treated for testicular torsion. J Androl 2007; 28: 528- 532

[5] Romeo C et al. Late hormonal function after testicular torsion. J Pediatr Surg 2010; 45: $411-413$

[6] Turner TT, Brown KJ. Spermatic Cord Torsion: Loss of spermatogenesis despite return of blood flow. Biol Reprod 1993; 49: 401 - 407

[7] Lotti F et al. Semen quality impairment is associated with sexual dysfunction according to its severity. Hum Reprod 2016; 31: 2668 2680 